\title{
Neuroprotective effects of dexmedetomidine on traumatic brain injury: Involvement of neuronal apoptosis and HSP70 expression
}

\author{
MAN-HE ZHANG ${ }^{1}$, XIU-MIN ZHOU ${ }^{2}$, JIAN-ZHONG CUI ${ }^{1}$, \\ KAI-JIE WANG ${ }^{2}$, YAN FENG ${ }^{1}$ and HONG-AO ZHANG ${ }^{2}$ \\ ${ }^{1}$ Department of Surgery, Hebei Medical University, Shijiazhuang, Hebei 050017; \\ ${ }^{2}$ Department of Neurosurgery, Tangshan Workers' Hospital, Tangshan, Hebei 063000, P.R. China
}

Received July 19, 2016; Accepted February 16, 2018

DOI: $10.3892 / \mathrm{mmr} .2018 .8898$

\begin{abstract}
The aim of the present study was to investigate the protective effect of dexmedetomidine (Dex) on traumatic brain injury (TBI), and further evaluate whether the underlying neuroprotective mechanisms are associated with neurological apoptosis and the expression of $70 \mathrm{kDa}$ heat shock protein (HSP70) in the hippocampus. A total of 90 adult male Sprague-Dawley rats were randomly assigned into 3 groups ( $n=30 /$ group): Sham, TBI and Dex groups. The rat models of TBI were established using a modified weight-drop device and Dex $(15 \mu \mathrm{g} / \mathrm{kg})$ was intravenously administered immediately following TBI. The brain edema and neurological function outcomes of TBI were assessed using wet-dry weight analysis and the Neurological Severity Score method. The expression levels of B-cell lymphoma-2 (Bcl-2) and Bcl-2-associated $\mathrm{X}$ protein (Bax) in the rat hippocampus were evaluated using immunohistochemical staining and western blot analysis. The protein levels of HSP70 in the hippocampal region were analyzed using western blot analysis. The results of the present study revealed that administration of Dex post-TBI improved brain edema and neurological outcomes, due to the attenuation of the TBI-induced reduction of Bax expression and increase of Bcl-2 and HSP70 expression. In conclusion, the results of the present study suggested that administration of Dex may serve as a neuroprotective agent against brain injury, at least partially via the inhibition of neuronal apoptosis and upregulation of HSP70 expression in the hippocampus.
\end{abstract}

Correspondence to: Dr Jian-Zhong Cui, Department of Surgery, Hebei Medical University, 361 East Zhongshan Road, Shijiazhuang, Hebei 050017, P.R. China

E-mail: jzcdoctor@163.com

Abbreviations: TBI, traumatic brain injury; Dex, dexmedetomidine; HSP70, 70 kDa heat shock proteins; SD, Sprague-Dawley

Key words: traumatic brain injury, dexmedetomidine, apoptosis, heat shock proteins, hippocampus, neuron

\section{Introduction}

Traumatic brain injury (TBI), a serious health condition that is increasing in prevalence, is one of the leading causes of mortality and long-term neurological disability worldwide $(1,2)$. Head injury, particularly in young men, is frequently caused by external physical forces, including falls, sport injuries, motor vehicle accidents and firearm incidents $(3,4)$. Impairment of cognitive, physical and psychosocial functions induced by TBI largely depends on the severity of the initial mechanical injury to the head and subsequent pathophysiological processes (5). It has been well established that the pathophysiology of TBI is a highly complex process that involves primary and secondary injury mechanisms (6). Primary injury, defined as the direct result of the mechanical effects of initial impact, can cause contusion, laceration, ischemia, diffuse axonal injury, diffuse swelling and intracranial hemorrhage at the moment of insult, which may result in neurological impairment (7). These effects are irreversible and can only be avoided via primary prevention strategies. Secondary injury is initiated by primary injury following the initial traumatic insult, or within h to days and even weeks post-injury $(8,9)$. A secondary insult can induce a progressive cascade of associated events, including inflammation, oxidative stress, calcium-mediated damage, neurotransmitter release, mitochondrial dysfunction (that subsequently triggers neuronal cell death), astrocyte proliferation and microglia activation (10-12). Secondary brain injury is thought to be responsible for the development of brain edema, neurological deficits and cognitive dysfunction following TBI (12). The delay in the induction of secondary injury provides a window for therapeutic TBI intervention. A series of biophysiological and pathological reactions following TBI contribute to subsequent neuronal cell death, including apoptosis, necrosis and necroptosis (13). Apoptosis, a programmed form of cell death, is implicated in pathological responses post-TBI, and contributes to secondary brain damage following TBI (14). Furthermore, rapid onset of cell death typically leads to tissue necrosis. Necrotic cell death is a consequence of acute disruption of cellular metabolism. In contrast to apoptosis, necrotic cell death rarely serves the requirements of the organism (15). A number of genes including Bcl-2 and Bax are known to regulate the apoptotic 
pathway, and the impairment of apoptosis is often caused by overexpression of the pro-survival protein Bcl-2 (16).

Determining the underlying pathological mechanisms and developing potential therapeutic strategies for TBI are major research areas that have attracted global attention. Despite the fact that numerous clinical and basic studies have focused on TBI treatment, there are limited pharmacological therapies available for the treatment of neurological injury and the prognosis for patients with TBI (17-19). Thus, an improved mechanistic understanding of TBI pathogenesis is required in order to develop therapeutic strategies for the treatment of TBI.

Dexmedetomidine (Dex), a highly selective $\alpha_{2}$-adrenoceptor agonist, previously provided neuroprotection against ischemia reperfusion-induced cerebral injury $(20,21)$, transient spinal ischemia (22) and isoflurane-induced neuroapoptosis (23). However, the underlying mechanisms of these processes have not been fully determined. In the present study, whether Dex possesses neuroprotective potential in a rat model of TBI was investigated. Furthermore, whether the TBI-associated neuroprotective effects of Dex are associated with neuronal apoptosis, and $70 \mathrm{kDa}$ heat shock protein (HSP70) protein expression levels in the hippocampus was investigated.

\section{Materials and methods}

Animals. A total of 90 adult male Sprague-Dawley (SD) rats, weighing 350-375 g (Vital River Laboratory Animal Technology Co., Ltd., Beijing, China) were used in the present study. The animals were housed with a controlled temperature (20-25 $\left.{ }^{\circ} \mathrm{C}\right)$ and humidity (50-65\%), a standard 12-h light/dark cycle, and had free access to food and water prior to and following the surgery/sham operation. All rats were sacrificed via cervical dislocation. All experimental procedures were carried out in accordance with the guidelines of the Chinese Council on Animal Protection (24) and were approved by the Animal Ethics Committee of Hebei Medical University (Hebei, China).

Experimental TBI model. The rat model of TBI was induced using a modified weight-drop device, as described previously by Marmarou et al (25). Briefly, rats were anaesthetised with intraperitoneal chloral hydrate $(40 \mathrm{mg} / \mathrm{kg})$. Following this, a midline incision was made in order to expose the skull between the bregma and lambda suture lines. Subsequently a steel disc (10 $\mathrm{mm}$ in diameter and $3 \mathrm{~mm}$ in thickness) was adhered to the skull using dental acrylic. Animals were then relocated onto a foam mattress placed underneath a weight-drop device and, following relocation, a weight of $450 \mathrm{~g}$ was dropped through a vertical tube from a height of $1.5 \mathrm{~m}$ onto the steel disc. Sham-operated animals underwent the same surgical procedure, however, they were not subjected to cortical impact. Following surgery, the wound was closed, and rats were then housed in individual cages and placed on heat pads $\left(37^{\circ} \mathrm{C}\right)$ for $24 \mathrm{~h}$ in order to maintain normal body temperature during the recovery period.

Group and drugs administration. The total 90 male SD rats were randomly divided into 3 groups ( $n=30 /$ group: Sham-operated, TBI and TBI treated with Dex groups. Animals in the TBI + Dex group received an intravenous injection of Dex (15 $\mu \mathrm{g} / \mathrm{kg}$; Jiangsu Hengrui Medicine Co., Ltd., Lianyungang, China) at $1 \mathrm{~h}$ post-TBI. The particular dose of Dex used in the present study was selected according to a previous study (26). Each group was then further divided into 3 subgroups ( $n=10 /$ subgroup); each subgroup of rats was sacrificed at either 12,24 or $72 \mathrm{~h}$ post-TBI, respectively.

Neurological function assessment. Neurobehavioral testing was performed using the Neurological Severity Score (NSS) assessment (24), which assesses motor, sensory, reflex and coordination abilities. One point was awarded for each failure to perform a particular task, and a score of 10 reflected maximal impairment. NSS was evaluated at 3 and 7 days post-TBI. An observer who was blinded to the group assignment of each rat, carried out the assessments. The difference between the initial NSS and that at any later time was calculated for each rat, and this value ( $\triangle \mathrm{NSS}$ ) was understood to reflect either the spontaneous recovery or the treatment-induced recovery of motor function.

Morris water maze test. The spatial learning ability of the rats was assessed using a Morris water maze as previously described (24). The Morris water maze consisted of a black circular pool (180 cm diameter, $45 \mathrm{~cm}$ high) filled with water (30 cm depth) at a temperature of $26^{\circ} \mathrm{C}$ and was virtually divided into 4 equivalent quadrants: North $(\mathrm{N})$, west $(\mathrm{W})$, south (S) and east (E). A $2 \mathrm{~cm}$ submerged escape platform (diameter $12 \mathrm{~cm}$, height $28 \mathrm{~cm}$; made opaque with paint) was placed in the middle of one of the quadrants equidistant from the sidewall and the center of the pool. Rats were trained to find the platform prior to either the TBI or sham operation. For each trial, the rat was randomly placed into a quadrant start point $(\mathrm{N}, \mathrm{S}, \mathrm{E}$ or $\mathrm{W})$ facing the wall of the pool and allowed a maximum of $60 \mathrm{sec}$ to find the escape platform. If the rat failed to escape within $90 \mathrm{sec}$, it was permitted to remain on the platform for a maximum of $20 \mathrm{sec}$ prior to returning to its holding cage for a new trial (intertrial interval, $20 \mathrm{sec}$ ). Maze performance was recorded using a video camera suspended above the maze and interfaced with a video tracking system (Huaibei Zhenghua Biological Instrument Co., Ltd., China). A total of 6 rats from each group were tested at day 3 and 6 different rats from each group were tested at day 7 (5 times each); rats tested on day 3 were not tested on day 7 and vice versa.

Investigation of brain edema. Brain edema was investigated via analysis of brain water content using the wet-dry weight method. Briefly, rats were anesthetized intraperitoneally using $10 \%$ chloral hydrate $(0.3 \mathrm{ml} / 100 \mathrm{~g})$ and sacrificed at $24 \mathrm{~h}$ post-TBI. The brains from rats in each group were rapidly separated and weighed, and then placed in an oven for $72 \mathrm{~h}$ at $100^{\circ} \mathrm{C}$. Following this, the brains were then reweighed in order to determine dry weight content. The percentage of water in the tissues was calculated according to the formula: Percent brain water $=[$ (wet weight-dry weight)/wet weight $] \times 100$ (27).

Immunohistochemical analysis. Immunohistochemical analysis was performed in accordance with the instructions of the StreptAvidin-Biotin Complex immunohistochemistry kit (Wuhan Boster Biological Engineering, Ltd., Wuhan, 
China). The brains were fixed in $4 \%$ paraformaldehyde for $24 \mathrm{~h}$ at room temperature, dehydrated by graded ethanol, and then embedded in paraffin. Following this, the sections $(5 \mu \mathrm{m})$ were then prepared and incubated with $3 \% \mathrm{H}_{2} \mathrm{O}_{2}$ for $10 \mathrm{~min}$ and $5 \%$ bovine serum albumin solution (Gibco; Thermo Fisher Scientific, Inc., Waltham, MA, USA) for $20 \mathrm{~min}$ at room temperature. Subsequently, sections were incubated overnight at $4{ }^{\circ} \mathrm{C}$ with rabbit anti-B-cell lymphoma-2 (Bcl-2; cat. no. sc-24511; 1:100) and anti-Bcl-2-associated X protein (Bax; cat. no. sc-4239; both Santa Cruz Biotechnology, Inc., Dallas, TX, USA; 1:100), and then with horseradish peroxidase (HRP)-conjugated anti-rabbit immunoglobulin (Ig)-G antibodies (Wuhan Boster Biotechnology Ltd., Wuhan, China; cat. no. SA1021; 1:3,000) for $40 \mathrm{~min}$ at room temperature. Following this, the detection reagent 3,3'-diaminobenzidine was used to visualize the immunohistochemical reaction under light microscope (BX53; Olympus, Tokyo, Japan). PBS was used as a substitute for the primary antibody in order to act as the negative control.

Immunofluorescence assay. After the experimental protocol, rats were anesthetized and sacrificed and the brain tissues were removed for immunofluorescence staining. The frozen sections $\left(-20^{\circ} \mathrm{C}, 15 \mu \mathrm{m}\right)$ were treated with $0.4 \%$ Triton- 100 (Wuhan Boster Biotechnology Ltd.) for $30 \mathrm{~min}$ and blocked in normal donkey serum (Wuhan Boster Biotechnology Ltd.) for $1.5 \mathrm{~h}$ at room temperature. The sections were then incubated in a mixture of rabbit anti-HSP70 polyclonal antibody (cat. no. sc-221731; 1:20), mouse anti-neuronal nuclei (NeuN; cat. no. sc-161127; 1:50; both Santa Cruz Biotechnology, Inc.) and mouse anti-GFAP (cat. no. sc-33673; 1:50; both Santa Cruz Biotechnology, Inc.) overnight at $4^{\circ} \mathrm{C}$. The following day, the sections were incubated in a mixture of fluorescein-conjugated anti-rabbit IgG (cat. no. sc-2357; 1:1,000) and anti-mouse IgG (cat. no. sc-2962.; 1:1,000; both Santa Cruz Biotechnology, Inc.) for $2 \mathrm{~h}$ at $37^{\circ} \mathrm{C}$ in the dark. The nuclei were then counterstained with DAPI for $15 \mathrm{~min}$ at room temperature. All microphotographs were taken under a laser scanning confocal microscope (Leica TCS SP8 STED 3X; serial no. 8100001247; Wetzlar, Germany, magnification, x1,000).

Western blot analysis. Brain tissues from damaged hippocampal regions in different groups at 12,24 or $72 \mathrm{~h}$ post-TBI were extracted and homogenized in RIPA lysis buffer (Wuhan Boster Biotechnology Ltd.). The lysates were centrifuged for $10 \mathrm{~min}$ at $12,000 \mathrm{x} \mathrm{g}, 4^{\circ} \mathrm{C}$, and the supernatants were transferred into a fresh tube. Total protein concentration was determined using the bicinchoninic acid reagent method. Samples (30 mg per sample) were separated on a $10 \%$ SDS-PAGE gel, and subsequently transferred to polyvinylidene fluoride membranes (Roche Diagnostics GmbH, Mannheim, Germany). The membranes were blocked with $10 \%$ non-fat milk (w/v) at room temperature for $2 \mathrm{~h}$ and then incubated overnight at $4^{\circ} \mathrm{C}$ with rabbit anti-Bax polyclonal antibodies (cat. no. sc-4239; 1:500), rabbit anti-Bcl-2 polyclonal antibodies (cat. no. sc-24511; 1:500), rabbit anti-HSP70 polyclonal antibodies (cat. no. sc-221731; $1: 500$ ) or rabbit anti- $\beta$-actin monoclonal antibodies (cat. no. sc-47778; all Santa Cruz Biotechnology, Inc.; dilution, 1:500). Membranes were then incubated with HRP-conjugated anti-rabbit IgG secondary antibody (cat. no. sc-2376; Santa Cruz Biotechnology, Inc.; dilution, 1:5,000) at room temperature for $1 \mathrm{~h}$. Immunoreactivity was detected using an enhanced chemiluminescence detection system (ChemiDoc XRS; Bio-Rad Laboratories, Inc., Hercules, CA, USA) and the densitometric signals were quantified using an Image Quant 5.2 software (GE Healthcare Life Sciences, Little Chalfont, UK). Protein expression was normalized to the intensity of corresponding bands for $\beta$-actin.

Statistical analysis. Data were expressed as the mean \pm standard error from three independent experiments. SPSS version 16.0 software (SPSS, Inc., Chicago, IL, USA) was used for statistical analysis. Statistical analysis was performed using one-way analysis of variance followed by the Student-Newman-Keuls post-hoc test, or the Student's t-test (for two means comparison). $\mathrm{P}<0.05$ was considered to indicate a statistically significant difference.

\section{Results}

Dex alleviates neurological deficits post-TBI. The NSS test was performed in order to investigate the long-term neurological function of rats post-TBI. The temporal alterations in functional recovery of the rat were expressed as $\triangle \mathrm{NSS}$. As revealed by the results of the NSS test presented in Fig. 1, TBI elicited a significant decline in neurological function, apparent according to the increase in $\triangle \mathrm{NSS}$ at 3 and 7 days post-TBI. However, Dex administration significantly decreased the neurological score in rats that underwent TBI, thereby suggesting that treatment with Dex post-TBI may improve recovery of neurological function.

Administration of Dex improves spatial learning and memory deficits post-TBI. The spatial learning and memory of rats was assessed using the Morris water maze at 3 and 7 days following TBI. As revealed by Fig. 2, all rats in the TBI group exhibited increased escape latency time periods as a result of their impaired ability to find the hidden platform compared with the sham group at 3 and 7 days. The rats subjected to Dex administration demonstrated a significant decrease in their escape latency time period compared with the rats belonging to the TBI group, thereby indicating that Dex administration may result in spatial learning and memory functional recovery post-TBI.

Administration of Dex attenuates brain edema post-TBI. The severity of brain edema was investigated by measuring the brain water content at set time points between $12 \mathrm{~h}$ and 7 days post-TBI. In the present study, the wet-dry weight method was used to determine brain water content. As demonstrated by Fig. 3, the water content of brain tissues post-TBI was significantly increased compared with the sham group. The administration of Dex markedly decreased brain water content compared with the TBI group; however, the brain water content still remained higher than that of the sham group.

Administration of Dex downregulates Bax expression post-TBI. At the $12 \mathrm{~h}$ post-TBI, Bax protein expression was measured using immunohistochemistry in the 


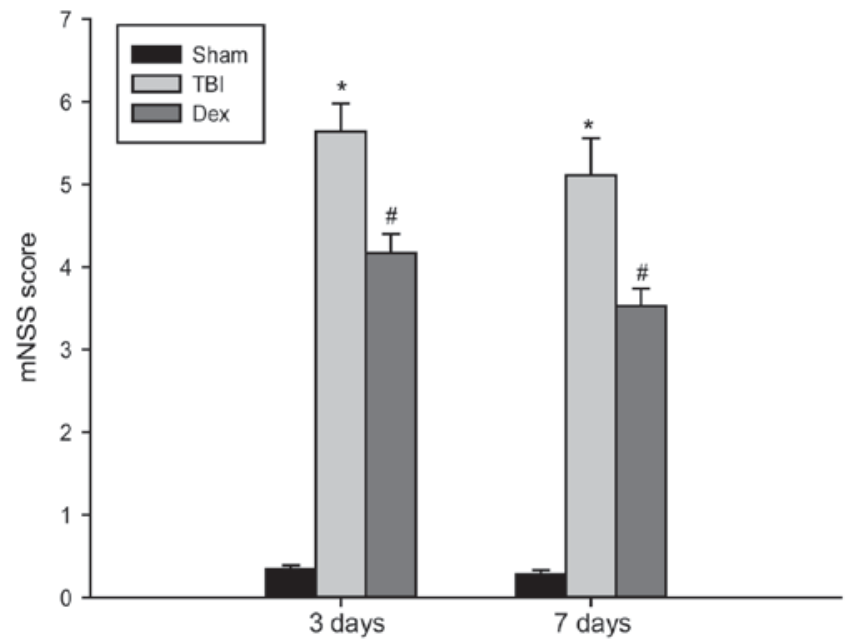

Figure 1. Effect of Dex administration on TBI-induced neurological deficits Following TBI, neurological function impairment was observed as revealed by an increase in $\triangle$ NSS. Dex treatment significantly improved neurological function. Bars represent mean \pm standard error. " $\mathrm{P}<0.05$ vs. sham group; ${ }^{\#} \mathrm{P}<0.05$ vs. TBI group. TBI, traumatic brain injury; Dex, dexmedetomidine; NSS, Neurological Severity Score.
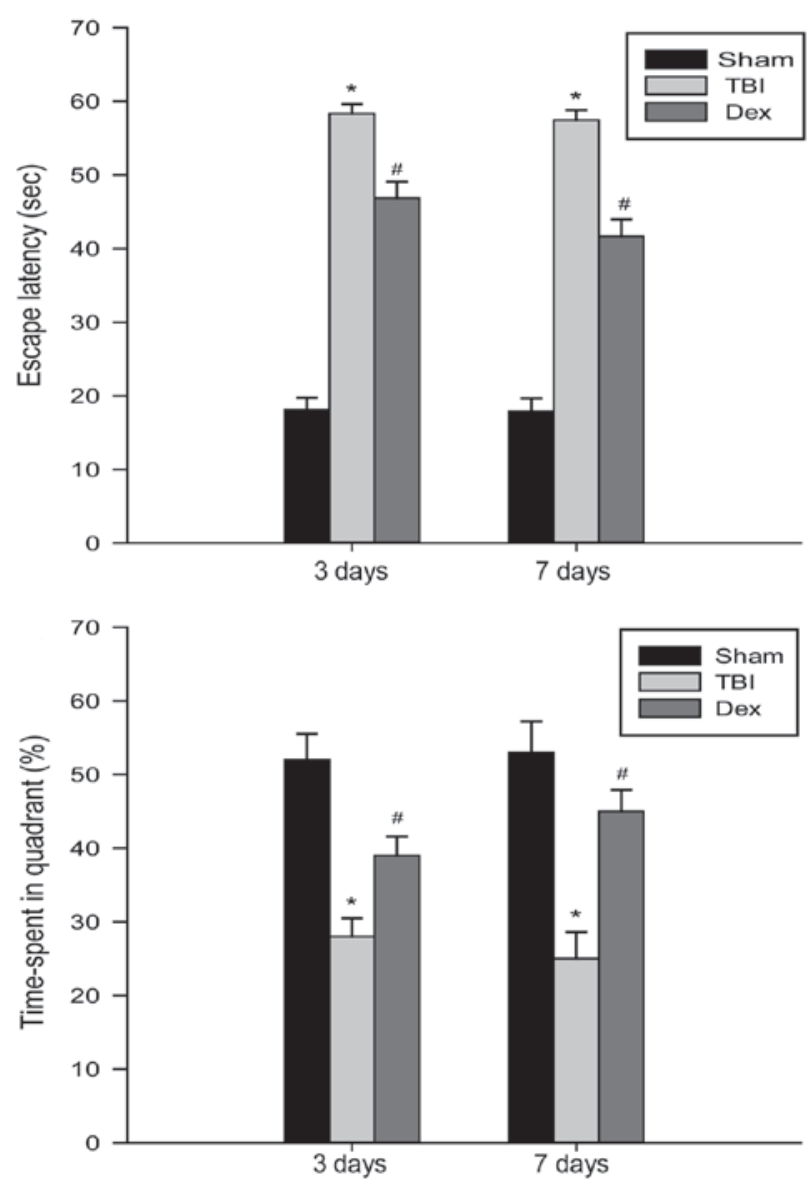

Figure 2. Effect of Dex administration on the escape latency performance and time spent in the quadrant was evaluated using a Morris water maze at 3 and 7 days following TBI. Bars represent the mean \pm standard error ( $\mathrm{n}=5$ /group). ${ }^{*} \mathrm{P}<0.05$ vs. sham group; ${ }^{\text {}} \mathrm{P}<0.05$ vs. TBI group. TBI, traumatic brain injury; Dex, dexmedetomidine.

paraffin-embedded brain tissue sections. Bax protein expression was revealed to be localized in the hippocampal neurons

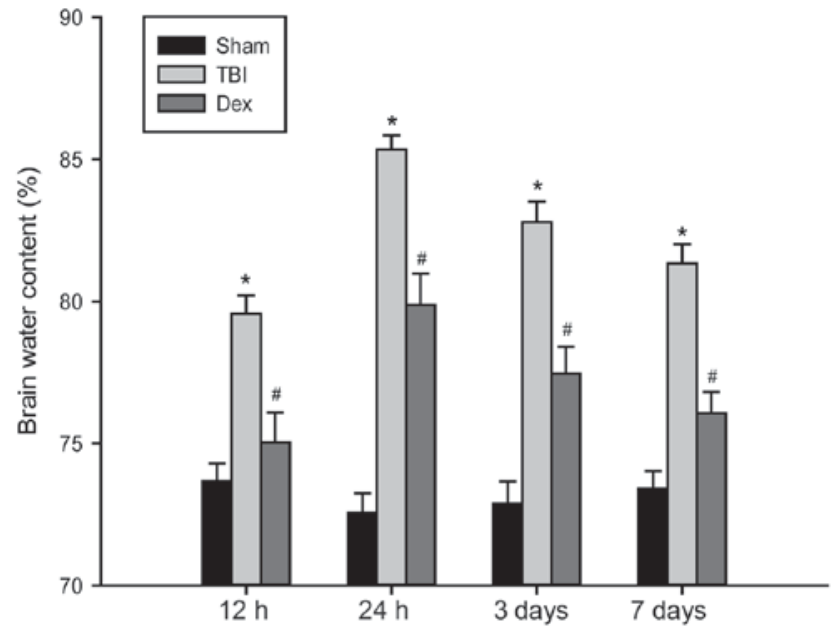

Figure 3. Effect of Dex administration on TBI-induced brain edema. Brain water content markedly increased at all time points, reaching a maximum level at $24 \mathrm{~h}$ post-injury. Treatment with Dex significantly decreased brain water content. Bars represent mean \pm standard error. ${ }^{*} \mathrm{P}<0.05$ vs. sham group; ${ }^{\#} \mathrm{P}<0.05$ vs. TBI group. TBI, traumatic brain injury; Dex, dexmedetomidine.

in all of rats. As demonstrated by Fig. 4A, Bax positive cells were observed within the hippocampus post-TBI; however, the administration of Dex post-TBI markedly reduced the number of Bax positive cells visualized. Following this, the expression of Bax protein was detected using western blot analysis. As revealed by Fig. 4B, basal Bax protein expression was low in the brains of the rats in the sham group. Compared with sham group rats, the level of Bax expression in the hippocampal fraction was significantly increased at $12 \mathrm{~h}$ post-TBI, reaching a maximum at $24 \mathrm{~h}$ post-TBI, which then steadily decreased until 7 days post-TBI. When compared with the TBI group, Bax protein expression levels were significantly reduced following Dex administration post-TBI. Taken together, these results suggested that Dex treatment may suppress the expression of Bax in the hippocampus following TBI.

Administration of Dex upregulates Bcl-2 expression post-TBI. Using immunohistological staining, the expression levels of Bcl-2 in samples of hippocampal brain tissue at different time intervals following TBI was investigated. As revealed by Fig. 5A, less Bcl-2 positive staining was visible in rats subjected to TBI when compared with the sham group. Furthermore, Fig. 5A revealed that rats treated with Dex demonstrated an increase in the number of Bcl-2 positive cells in the injured hippocampus compared with the TBI group. Following this, western blot analysis was performed in order to determine Bcl-2 protein expression levels. As demonstrated in Fig. 5B, basal expression of Bcl-2 was detected in the hippocampus samples from the sham group. Compared with the sham group, the expression of Bcl-2 was significantly reduced in the TBI group $12 \mathrm{~h}$ post-injury and reached a minimum concentration at $24 \mathrm{~h}$ post-TBI. However, Bcl-2 protein expression level in the hippocampal tissue was significantly increased in the Dex group when compared with the TBI group. Taken together, these results indicated that the administration of Dex may upregulate the expression of Bcl-2 in the hippocampus following TBI. 
A

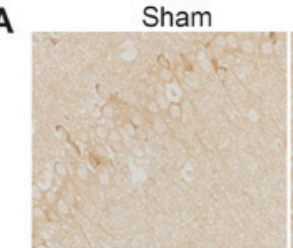

B
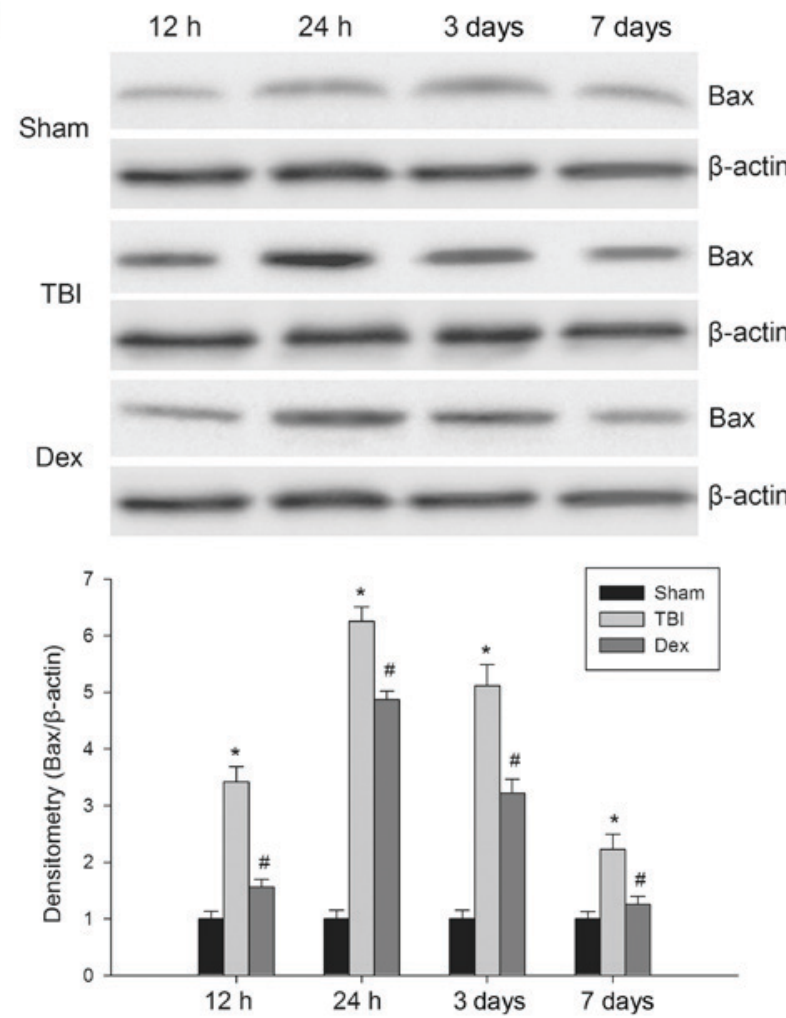

Figure 4. Effect of Dex administration on Bax protein expression post-TBI. (A) Representative images of immunohistochemical staining of Bax at $24 \mathrm{~h}$ post-TBI, magnification, $x$ 400. (B) Western blot analysis of Bax expression in the hippocampus and densitometry analysis of Bax bands corresponding to $\beta$-actin. Treatment with Dex attenuated the otherwise TBI-induced increase in Bax protein expression. Bars represent mean \pm standard error. ${ }^{*} \mathrm{P}<0.05$ vs. sham group; ${ }^{\#} \mathrm{P}<0.05$ vs. TBI group. TBI, traumatic brain injury; Dex, dexmedetomidine; Bax, B-cell lymphoma 2-associated X protein.

Administration of Dex enhances HSP70 expression post-TBI. In order to determine whether Dex protects against TBI via the modulation of HSP70 expression, the expression levels of HSP70 in the hippocampus of the different experimental rat groups were investigated. Firstly, the co-expression of HSP70 and either NeuN (Fig. 6) or GFAP (Fig. 7) in the hippocampus of rats was observed using a laser scanning confocal microscope. It was revealed that $\mathrm{HSP} 70$ primarily presented itself in the neurons of the hippocampal CA1 region. Additionally, the protein expression levels of HSP70 in the hippocampus at 12,24 h, 3 and 7 days post-TBI were investigated using western blot. As demonstrated in Fig. 8, HSP70 expression levels were significantly higher in the TBI group compared with the sham group and peaked at $24 \mathrm{~h}$ post-TBI. Furthermore, Dex treatment significantly enhanced the protein expression of HSP70 post-TBI (Fig. 8).

\section{Discussion}

Apoptosis, the morphological manifestation of programmed cell death occurring under physiological and pathological
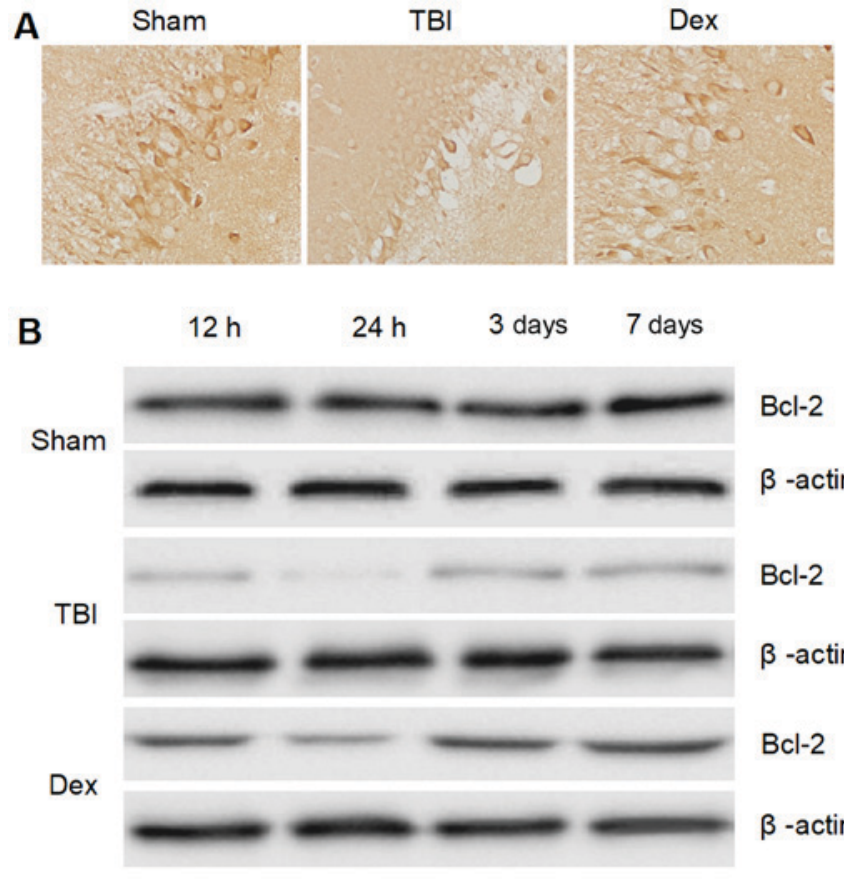

$\mathrm{Bcl}-2$

$\beta$-actin

Bcl-2

$\beta$-actin

$\mathrm{Bcl}-2$

$\beta$-actin

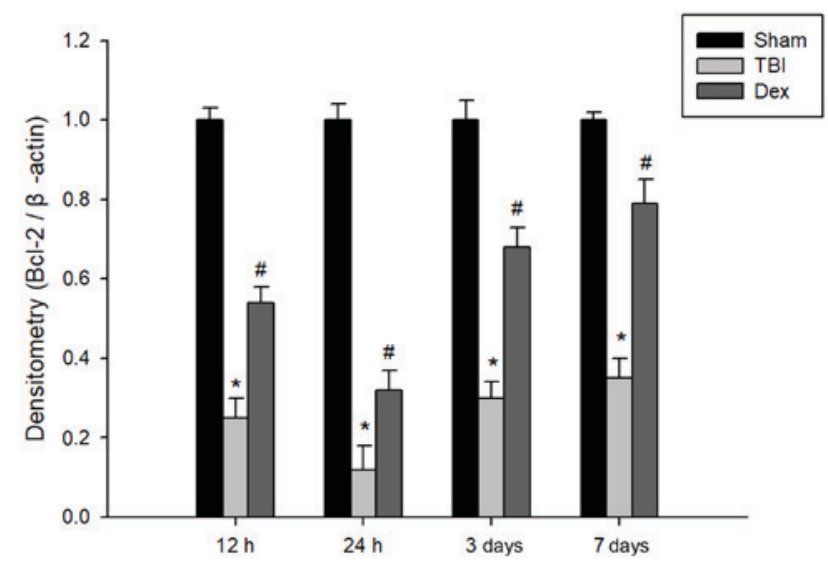

Figure 5. Effect of Dex on Bcl-2 protein expression post-TBI. (A) Representative images of immunohistochemical staining of Bcl-2 at $24 \mathrm{~h}$ post-TBI, magnification $\mathrm{x} 400$. (B) Western blot analysis of Bcl-2 expression in the hippocampus and densitometry analysis of $\mathrm{Bcl}-2$ bands corresponding to $\beta$-actin. Treatment with Dex attenuated the otherwise TBI-induced decrease in Bcl-2 protein expression. Bars represent mean \pm standard error. ${ }^{*} \mathrm{P}<0.05$ vs. sham group; ${ }^{\text {"}} \mathrm{P}<0.05$ vs. TBI group. TBI, traumatic brain injury; Dex, dexmedetomidine; Bcl-2, B-cell lymphoma 2.

conditions, is characterized by DNA fragmentation, chromatin compaction, nuclear shrinkage and cytoplasmic condensation (28). TBI triggers a complex cascade of apoptotic events, which contribute to neuronal damage and neurological dysfunction (29). The hippocampus, a key brain structure associated with functional impairments, is particularly vulnerable to brain injury. These early changes of TBI occur in the hippocampal region (30).

The aim of the present study was to investigate brain edema, behavioral impairment and neuronal apoptosis in the hippocampus following experimental TBI. A rat model of TBI utilizing a simple weight-drop device was established, and this simple model was capable of producing a graded brain injury in the rodent without initiating hypertensive surge or excessive brain-stem damage. The results of the present study 

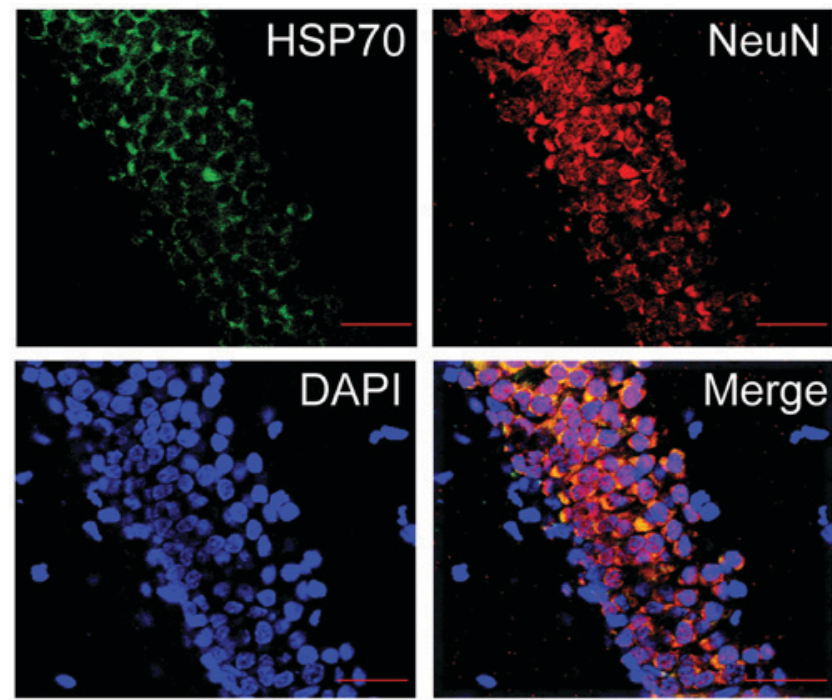

Figure 6. Colocalization of HSP70 in the neurons of rat hippocampal CA1 region, with NeuN staining. All microphotographs were visualized using confocal laser scanning microscopy. Scale bars $=50 \mu \mathrm{m}$. HSP70, heat shock protein; NeuN, neuronal nuclei.
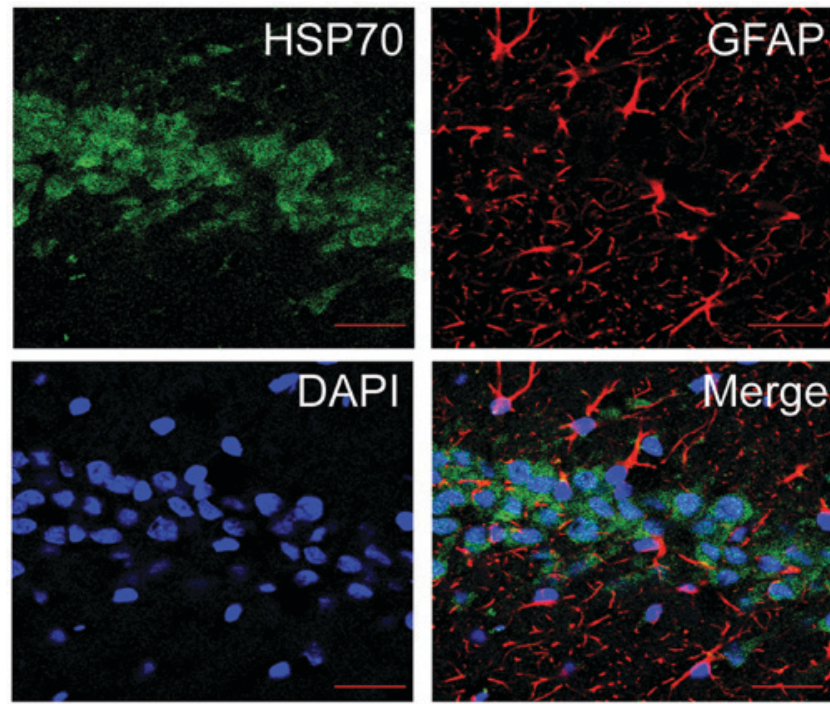

Figure 7. Colocalization of HSP70 in the astrocytes of rat hippocampal CA1 region, with GFAP staining. All microphotographs were visualized using confocal laser scanning microscopy. Scale bars $=20 \mu \mathrm{m}$. HSP70, heat shock protein; GFAP, glial fibrillary acidic protein.

demonstrated that the brain water content and neurological deficit scores of rats subjected to TBI were significantly lower at $24 \mathrm{~h}$ post-TBI than those demonstrated by sham group.

Members of the Bcl-2 family are important regulators of cellular apoptosis. The Bax protein promotes neuronal apoptosis via direct activation of proapoptotic factors and inactivation of antiapoptotic Bcl-2 family members (31). By contrast, the $\mathrm{Bcl}-2$ protein inhibits apoptosis and is implicated in neuroprotective events post-TBI. The ratio of Bcl-2 and Bax expression determines the extent of cellular apoptosis. In addition, HSP70, a highly conserved molecular chaperone, confers cellular protection against insults as it possesses potent anti-apoptotic properties (32). In the present study, the expression levels of Bax, $\mathrm{Bcl}-2$ and

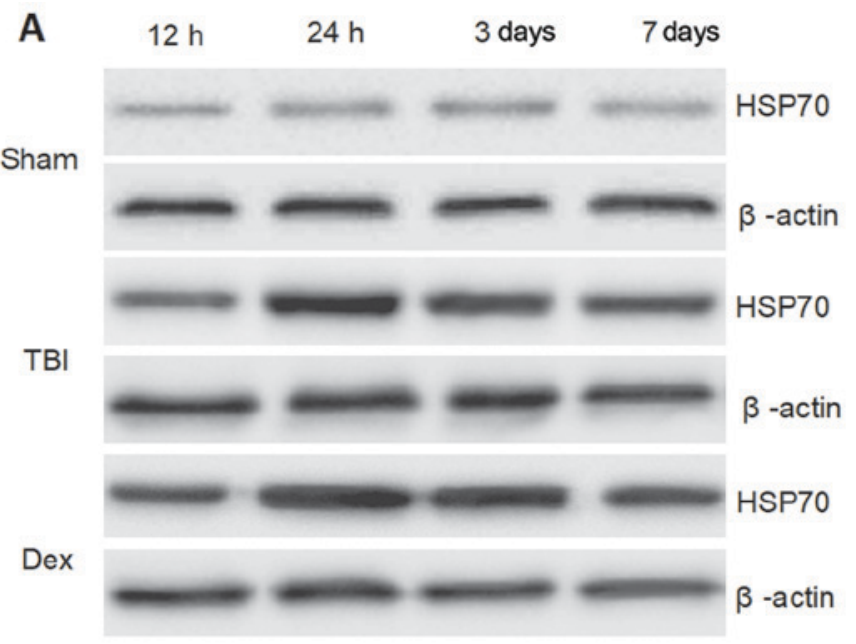

B

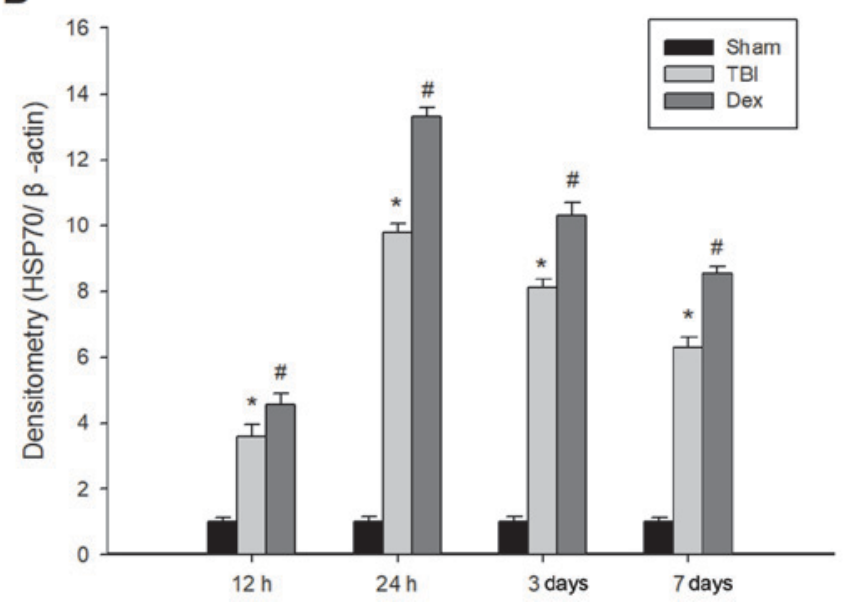

Figure 8. Effect of Dex administration on HSP70 protein expression. (A) The results presented are of the western blot analysis of HSP70 protein expression in the hippocampus and (B) densitometry analysis of HSP70 bands corresponding to $\beta$-actin. Dex administration was revealed to increase HSP70 expression post-TBI compared with non-treated TBI rats. Bars represent mean \pm standard error. ${ }^{*} \mathrm{P}<0.05$ vs. sham group; ${ }^{\prime} \mathrm{P}<0.05$ vs. TBI group. TBI, traumatic brain injury; Dex, dexmedetomidine; HSP70, heat shock protein.

HSP70 in the hippocampus post-TBI were determined by immunohistochemistry and western blot analysis. It was revealed that Bc1-2 expression decreased post-TBI, while the expression of Bax and HSP70 increased. These results suggested that neuronal apoptosis may be implicated in the pathophysiological process of TBI. Taken together, these results suggested that TBI may initiate a neuronal apoptosis cascade that contributes to substantial neuronal damage, brain edema and behavioral impairment.

The results of the present study suggested that anti-apoptotic proteins may serve as potential therapeutic targets for the protection of the damaged brain in cases of TBI. Previous clinical and experimental studies have demonstrated that the administration of Dex is an effective intervention strategy for several neurological diseases (33-35). Furthermore, it has recently been reported that Dex has the ability to pass freely across the blood brain barrier, and its metabolism predominantly occurs in the liver (33). In addition, a further recent study demonstrated that patients with liver disease have a significantly decreased clearance rate of Dex (36). A large 
proportion of experimental data has suggested that Dex is critically implicated in ischemic/reperfusion models in different organs, including the brain (21), heart (37), kidney (38) and lung (39). Furthermore, Dex has been extensively studied due to its wide range of modulatory activities, including modulation of inflammatory processes, gene expression, oxidative stress reaction, transmitter release and channel activation (40). In the present study, the determination of the function of Dex in the pathophysiological process of brain damage, cerebral edema and neurological function impairment was investigated post-TBI in comparison with the sham group at $24 \mathrm{~h}$ post-TBI. The results revealed that Dex administration markedly attenuated the brain water content post-TBI. In addition, following Dex treatment for TBI, the $\triangle$ NSS was significantly lower than that of non-treated rats at $24 \mathrm{~h}$ post-TBI. A previous study demonstrated that Dex has been confirmed to confer neuroprotection for rats subjected to weight-drop contusion injury (41). However, the exact mechanisms underlying this neuroprotective process remain undetermined.

The present study then investigated the effects of Dex on neuronal apoptosis and HSP70 protein expression at 12 , 24 and $72 \mathrm{~h}$ post-TBI. The results indicated that the administration of Dex had the potential to attenuate the otherwise increased expression of Bax and reduced expression of Bcl-2 levels, and to enhance the expression of HSP70 in the injured hippocampus following TBI. These results suggested that Dex may possess a neuroprotective function and thus in turn, may be a potent therapeutic agent for TBI treatment. The results also indicated that the underlying mechanisms of this process may be associated with the modulation of neuronal apoptosis. Therefore, Dex may serve as a novel therapeutic target for the treatment of TBI.

In conclusion, the results of the present study suggested that the administration of Dex post-TBI may improve clinical outcomes by reducing brain edema and neurological functional impairment via anti-apoptotic mechanisms involving the modulation of apoptosis-associated proteins $\mathrm{Bax}, \mathrm{Bcl}-2$ and HSP70 expression levels. This may be an important mechanism through which Dex provides an important neuroprotective effect following TBI. Thus, administration of Dex may be considered as a candidate for clinical trials investigating alternative treatments for patients with TBI. For future study, potential cellular and molecular mechanisms implicated in the neuroprotective effect of Dex administration, as well as signal transduction pathways involved in this process, should be investigated.

\section{Acknowledgements}

The present study was supported by a grant of Natural Science Foundation of Hebei Province. The authors would like to thank Junling Gao who provided technical help and writing assistance, as well as Yanxia Tian who contributed certain reagents and provided technical support.

\section{Funding}

The present study was supported by a grant from the Natural Science Foundation of Hebei Province (grant nos. H2012401071 and 2014105079).

\section{Availability of data and materials}

All data generated or analyzed during this study are included in this published article.

\section{Authors' contributions}

MZ made substantial contributions to conception, design, acquisition of data, analysis and interpretation of data, was responsible for writing and revision of the manuscript. JC contributed to the conception, design, writing, and revision of the manuscript. YF and $\mathrm{HZ}$ were responsible for acquisition of data, or analysis and interpretation of data. XZ and KW carried out the experiments. All authors read and approved the final manuscript.

\section{Ethics approval and consent to participate}

All experimental procedures were approved by the Animal Ethics Committee of Hebei Medical University (Hebei, China).

\section{Consent for publication}

Not applicable.

\section{Competing interests}

The authors declare that they have no competing interests.

\section{References}

1. Cheng G, Fu L, Zhang HY, Wang YM, Zhang LM and Zhang JN: The role of mitochondrial calcium uniporter in neuroprotection in traumatic brain injury. Med Hypotheses 80: 115-117, 2013.

2. Kumar A and Loane DJ: Neuroinflammation after traumatic brain injury: Opportunities for therapeutic intervention. Brain Behav Immun 26: 1191-1201, 2012.

3. Summers CR, Ivins B and Schwab KA: Traumatic brain injury in the United States: An epidemiologic overview. Mt Sinai J Med 76: 105-110, 2009.

4. Stein SC, Georgoff P, Meghan S, Mizra K and Sonnad SS: 150 years of treating severe traumatic brain injury: A systematic review of progress in mortality. J Neurotraum 27: 1343-1353, 2010.

5. Smania N, Avesani R, Roncari L, Ianes P, Girardi P, Varalta V, Gambini MG, Fiaschi A and Gandolfi M: Factors predicting functional and cognitive recovery following severe traumatic, anoxic and cerebrovascular brain damage. J Head Trauma Rehab 28: 131-140, 2013.

6. Weber JT: Altered calcium signaling following traumatic brain injury. Front Pharmacol 3: 60, 2012.

7. Harvey LA and Close JC: Traumatic brain injury in older adults: Characteristics, causes and consequences. Injury 43: 1821-1826, 2012.

8. Mangat HS: Severe traumatic brain injury. Continuum (Minneap Minn) 18: 532-546, 2012.

9. Zink BJ, Szmydynger-Chodobska J and Chodobski A: Emerging concepts in the pathophysiology of traumatic brain injury. Psychiatr Clin North Am 33: 741-756, 2010.

10. Hart T, Brenner L, Clark AN, Bogner JA, Novack TA, Chervoneva I, Nakase-Richardson R and Arango-Lasprilla JC: Major and minor depression after traumatic brain injury. Arch Phys Med Rehabi 92: 1211-1219, 2011.

11. Bye N, Carron S, Han X, Agyapomaa D, Ng SY, Yan E, Rosenfeld JV and Morganti-Kossmann MC: Neurogenesis and glial proliferation are stimulated following diffuse traumatic brain injury in adult rats. J Neurosci Res 89: 986-1000, 2011.

12. Loane DJ and Faden AI: Neuroprotection for traumatic brain injury: Translational challenges and emerging therapeutic strategies. Trends Pharmacol Sci 31: 596-604, 2010. 
13. Blennow K, Hardy $\mathrm{J}$ and Zetterberg $\mathrm{H}$ : The neuropathology and neurobiology of traumatic brain injury. Neuron 76: 886-899, 2012.

14. Xu X, Jiang R, Gong P, Liu Q, Chen Y, Hou S, Yuan D, Shi J and Lan Q: Up-regulation of FOS-like antigen 1 contributes to neuronal apoptosis in the cortex of rat following traumatic brain injury. Metab Brain Dis 33: 115-125, 2018.

15. Sato A: Novel anticancer strategy targeting switch mechanisms in two types of cell death: Necrosis and apoptosis. Yakugaku Zasshi: 1315-1321, 2017.

16. Anderson MA, Huang D and Roberts A: Targeting BCL2 for the treatment of lymphoid malignancies. Semin Hematol 51: 219-227, 2014.

17. Hartings JA, Bullock MR, Okonkwo DO, Murray LS, Murray GD, Fabricius M, Maas AI, Woitzik J, Sakowitz O, Mathern B, et al: Spreading depolarisations and outcome after traumatic brain injury: A prospective observational study. Lancet Neurol 10: 1058-1064, 2011.

18. Andriessen TM,Horn J,Franschman G, van der Naalt J, Haitsma I, Jacobs B, Steyerberg EW and Vos PE: Epidemiology, severity classification and outcome of moderate and severe traumatic brain injury: A prospective multicenter study. J Neurotraum 28 : 2019-2031,2011.

19. Zhang J, Jiang R, Liu L, Watkins T, Zhang F and Dong JF: Traumatic brain injury-associated coagulopathy. J Neurotraum 29: 2597-2605, 2012.

20. Wang Z, Kou D, Li Z, He Y, Yu W and Du H: Effects of propofol-dexmedetomidine combination on ischemia reperfusion-induced cerebral injury. NeuroRehabilitation 35: 825-834, 2014.

21. Zhu YM, Wang CC, Chen L, Qian LB, Ma LL, Yu J, Zhu MH, Wen CY, Yu LN and Yan M: Both PI3K/Akt and ERK1/2 pathways participate in the protection by dexmedetomidine against transient focal cerebral ischemia/reperfusion injury in rats. Brain Res 1494: 1-8, 2013

22. Goyagi T and Tobe Y: Dexmedetomidine improves the histological and neurological outcomes $48 \mathrm{~h}$ after transient spinal ischemia in rats. Brain Res 1566: 24-30, 2014.

23. Liao Z, Cao D, Han X, Liu C, Peng J, Zuo Z, Wang F and Li Y: Both JNK and P38 MAPK pathways participate in the protection by dexmedetomidine against isoflurane-induced neuroapoptosis in the hippocampus of neonatal rats. Brain Res Bull 107: 69-78, 2014

24. Cui C, Cui Y, Gao J, Sun L, Wang Y, Wang K, Li R, Tian Y, Song S and Cui J: Neuroprotective effect of ceftriaxone in a rat model of traumatic brain injury. Neurol Sci 35: 695-700, 2014

25. Marmarou A, Foda MA, van den Brink W, Campbell J, Kita H and Demetriadou K: A new model of diffuse brain injury in rats. Part I: Pathophysiology and biomechanics. J Neurosurg 80 291-300, 1994.

26. Yang NL, Xu DM and Ming GF: Intervention effect of dexmedetomidine on inflammatory response following traumatic brain injury in rats. Pract Prev Med 17: 243-245, 2010.

27. Roof RL, Duvdevani R, Heyburn JW and Stein DG: Progesterone rapidly decreases brain edema: Treatment delayed up to $24 \mathrm{~h}$ is still effective. Exp Neurol 138: 246-251, 1996.
28. Zhang X, Chen Y, Jenkins LW, Kochanek PM and Clark RS: Bench-to-bedside review: Apoptosis/programmed cell death triggered by traumatic brain injury. Crit Care 9: 66-75, 2005.

29. Stoica BA and Faden AI: Cell death mechanisms and modulation in traumatic brain injury. Neurotherapeutics 7: 3-12, 2010.

30. Fanselow MS and Dong HW: Are the dorsal and ventral hippocampus functionally distinct structures? Neuron 65: 7-19, 2010.

31. Shamas-Din A, Brahmbhatt H, Leber B and Andrews DW: BH3-only proteins: Orchestrators of apoptosis. Biochim Biophys Acta 1813: 508-520, 2011.

32. Feder ME and Hofmann GE: Heat-shock proteins, molecular chaperones and the stress response: Evolutionary and ecological physiology. Annu Rev Physiol 61: 243-282, 1999.

33. Xiong B, Shi QQ and Miao CH: Dexmedetomidine renders a brain protection on hippocampal formation through inhibition of nNOS-NO signalling in endotoxin-induced shock rats. Brain Inj 28: 1003-1008, 2014

34. Wang X, Ji J, Fen L and Wang A: Effects of dexmedetomidine on cerebral blood flow in critically ill patients with or without traumatic brain injury: A prospective controlled trial. Brain Inj 27: 1617-1622, 2013.

35. James ML, Olson DM and Graffagnino C: A pilot study of cerebral and haemodynamic physiological changes during sedation with dexmedetomidine or propofol in patients with acute brain injury. Anaesth Intens Care 40: 949-957, 2012.

36. Nasrallah N A, Thomas M, Kuehl S and Diab K: The use of dexmedetomidine for longer than $48 \mathrm{~h}$ with evaluation of its secondary outcome in patients with liver disease. Chest 150: 228A, 2016

37. Guler L, Bozkirli F, Bedirli N, Unal Y, Guler A, Oztas Y, Balta S, Cakar M, Demirkol S, Arslan Z and Unlu M: Comparison of the effects of dexmedetomidine vs. ketamine in cardiac ischemia/reperfusion injury in rats-preliminary study. Adv Clin Exp Med 23: 683-689, 2014

38. Si YN, Bao HG, Xu L, Wang XL, Shen Y, Wang JS and Yang XB Dexmedetomidine protects against ischemia/reperfusion injury in rat kidney. Eur Rev Med Pharmaco Sci 18: 1843-1851, 2014.

39. Jiang L, Li L, Shen J, Qi Z and Guo L: Effect of dexmedetomidine on lung ischemiareperfusion injury. Mol Med Rep 9: 419-426, 2014.

40. Cai Y, Xu H, Yan J, Zhang L and Lu Y: Molecular targets and mechanism of action of dexmedetomidine in treatment of ischemia/reperfusion injury. Mol Med Rep 9: 1542-1550, 2014.

41. Shen M, Wang S, Wen X, Han XR, Wang YJ, Zhou XM, Zhang MH, Wu DM, Lu J and Zheng YL: Dexmedetomidine exerts neuroprotective effect via the activation of the $\mathrm{PI} 3 \mathrm{~K} / \mathrm{Akt} / \mathrm{mTOR}$ signaling pathway in rats with traumatic brain injury. Biomed Pharmacother 95: 885-893, 2017.

(i) $(-)$ This work is licensed under a Creative Commons Attribution-NonCommercial-NoDerivatives 4.0 International (CC BY-NC-ND 4.0) License. 